\title{
Hospitalization for lower respiratory disease during 20 yrs among under 5 yr old children in Stockholm County: a population based survey
}

\author{
M. Wickman*+, B.Y. Farahmand ${ }^{\ddagger}$, P-G. Persson ${ }^{\ddagger}$, G. Pershagen* ${ }^{\S}$
}

Hospitalization for lower respiratory disease during 20 yrs among under 5 yr old children in Stockholm County: a population based survey. M. Wickman, B.Y. Farahmand, P-G. Persson, G. Pershagen. (CERS Journals Ltd 1998.

ABSTRACT: Lower respiratory disease (LRD) is a common cause of hospitalization in infants, and episodes of obstructive LRD increase the risk for asthma later in life. The purpose of this study was to assess time trends and geographical variation of first time hospitalization for LRD among children in Stockholm County, Sweden.

Data on first time admittance for LRD among children aged up to 5 yrs from 1973 through 1992 were obtained from the Stockholm County Council hospital discharge register, and population register data were used for estimation of the population at risk. Municipal data were available for 1982-1992 on outbreaks of respiratory syncytial virus (RSV) infections and socio-economic factors.

A total of 12,450 children had been hospitalized for the first time with LRD. For children aged $<2$ yrs a $100 \%$ increase in the rate of first time hospitalization for LRD was observed during the study period and children aged up to $1 \mathrm{yr}$ predominated $(50 \%)$. The average yearly increase in the population based first time hospital admission rate for LRD was $1.82 \%$. Males constituted $65 \%$ of the cases. Of the diagnoses, 89.6\% were consistent with obstructive LRD, e.g. asthma and obstructive bronchitis. In children aged up to $1 \mathrm{yr}$, peaks in the rate of hospitalization tended to coincide with outbreaks of RSV infections. Differences in hospitalization between municipalities seemed to be partly explained by differences in the admission practices of the four paediatric hospitals in the area.

We conclude that the increased rate of hospitalization for lower respiratory disease in infants of Stockholm County may reflect a true increase in the incidence of obstructive respiratory disease. However, several factors determine admittance to hospital and the results need to be interpreted with caution.

Eur Respir J 1998; 11: 366-370.

Lower respiratory disease (LRD) with obstructive symptoms (i.e. asthma and wheezing bronchitis) is one of the most common causes of hospitalization in early childhood [1]. Obstructive LRD in infancy is associated with asthma in childhood [2]. The occurrence of childhood asthma and hospital admissions for obstructive respiratory disease has been reported to increase in many Western countries during recent decades [3-5], although, the evidence is not consistent [6].

A number of risk factors for obstructive LRD besides heredity have been identified in children, such as viral infections [7-9], environmental tobacco smoke [10-14], damp and cramped housing [15-17] and socio-economic factors [18], whereas breast-feeding seems to be protective [19-22].

In this study we report time trends and geographic differences in rates of first time hospitalization for LRD among infants in Stockholm County during a period of 20 yrs. In addition, data were obtained on some risk factors for LRD in children, such as respiratory syncytial virus (RSV) infections.
*Dept of Environmental Health, Stockholm County Council, ${ }^{+}$Dept of Paediatrics, St. Göran's Children's Hospital, †Dept of Epidemiology, Stockholm County Council, and ${ }^{\S}$ Institute of Environmental Medicine, Karolinska Institute, Stockholm, Sweden.

Correspondence: M. Wickman

Dept of Environmental Medicine

Norrbacka Building, level 3

Karolinska Hospital

S-171 76 Stockholm

Sweden

Fax: 46851777900

Keywords: Asthma

childhood

hospitalization

respiratory disease

Received: November 251996

Accepted after revision September 281997

\section{Methods}

Children aged $<5$ yrs who had been admitted for the first time to any hospital in the county of Stockholm with diagnoses consistent with LRD during 1973-1992 were identified from the hospital discharge register of the Stock-holm County Council. This is a computerized register containing all hospital admissions in Stockholm County. An individual identification number was used to identify the persons in this register. Until 1986 the diagnoses includ- ed bronchial asthma, bronchiolitis, obstructive bronchitis and viral pneumonia according to the International Classification of Disease (ICD)-8: 466, 470.00, 480.00, 490.99 and 493.00. From 1987 ICD-9 was used: 466, 480, 490 and 493. The population register of Stockholm County provided information for estimation of the population at risk. The population of Stockholm County was 1.48 million in 1970 and 1.67 million in January 1993 with a gradual increase of the population aged $<5$ yrs from around 90,000 at the beginning of the 1980s to 117,452 at the end of the study period. 
Table 1. - Number of children aged 0-4 yrs admitted to hospital for the first time due to lower respiratory disease in Stockholm County during 1973-1992

\begin{tabular}{lrrrrrr}
\hline & \multicolumn{7}{c}{ Age yrs } \\
\cline { 2 - 7 } & 0 & 1 & 2 & 3 & 4 & $0-4$ \\
\hline 1973-1981 & & & & & & \\
Boys n & 1505 & 855 & 404 & 228 & 190 & 3182 \\
Girls n & 854 & 496 & 240 & 138 & 122 & 1850 \\
Total n & 2359 & 1351 & 644 & 366 & 312 & 5032 \\
1982-1992 & & & & & & \\
Boys n & 2434 & 1283 & 569 & 301 & 158 & 4754 \\
Girls n & 1388 & 710 & 321 & 154 & 100 & 2673 \\
Total n & 3822 & 1993 & 890 & 455 & 258 & 7418 \\
1973-1992 & & & & & & \\
Boys n & 3939 & 2138 & 973 & 529 & 348 & 7927 \\
Girls n & 2242 & 1206 & 561 & 292 & 222 & 4523 \\
Total n & 6181 & 3344 & 1534 & 821 & 570 & 12450 \\
\hline
\end{tabular}

During the observation period, 12,450 children aged $<5$ yrs were admitted for the first time for LRD at any of the hospitals of Stockholm county (table 1). The four children's hospitals within the county had $97.7 \%$ of the admittances for LRD.

The county of Stockholm has 25 municipalities and from 1982 data were available on a municipal level. During 1982-1992, 87 of the children hospitalized for LRD had resided outside Stockholm and residential data were missing for another 213 children. These 300 children (4\%) were excluded from analyses of municipal data. Data on average income, population density, educational level and number of immigrants were obtained for each municipality. The Swedish Institute for Infectious Disease Control provided information on epidemic outbreaks of respiratory infections caused by RSV. Data on number of available beds and duration of each admission were collected from the Stockholm County Council regarding the four children's hospitals in Stockholm during the period 19821992. A total of 101,530 children aged $<5$ yrs had been hospitalized at any of these hospitals during the relevant period and 11,160 of the children hospitalized for LRD of which 7,205 admissions were for the first time.

The relative risk (RR) of first time hospitalization for LRD in the population of children aged $<5$ yrs living in Stockholm County was calculated in relation to gender, age, calendar year and municipality using the rate for females, children aged $<1 \mathrm{yr}, 1973$ and the municipality of Stockholm City as reference. Analyses were performed with the GLIM statistical package (Royal Statistical Society, London, UK) [23]. Maximum-likelihood estimates of the RR were obtained by the multiplicative Poisson model [24]. For analysis of the time trend, this model takes into account changes in the age (by five age categories $0,1,23$, and 4 yrs) and gender distribution during the study period. The comparisons of catchment areas were adjusted for calendar year in addition to age by $1 \mathrm{yr}$ age groups and gender. The standard deviations of the RR estimates were used for determination of the $95 \%$ confidence interval $(95 \% \mathrm{CI})$.

\section{Results}

Fluctuations of the hospitalization rate for LRD in children aged $<5$ yrs were seen during the study period, but there seemed to be an overall increase during this period.

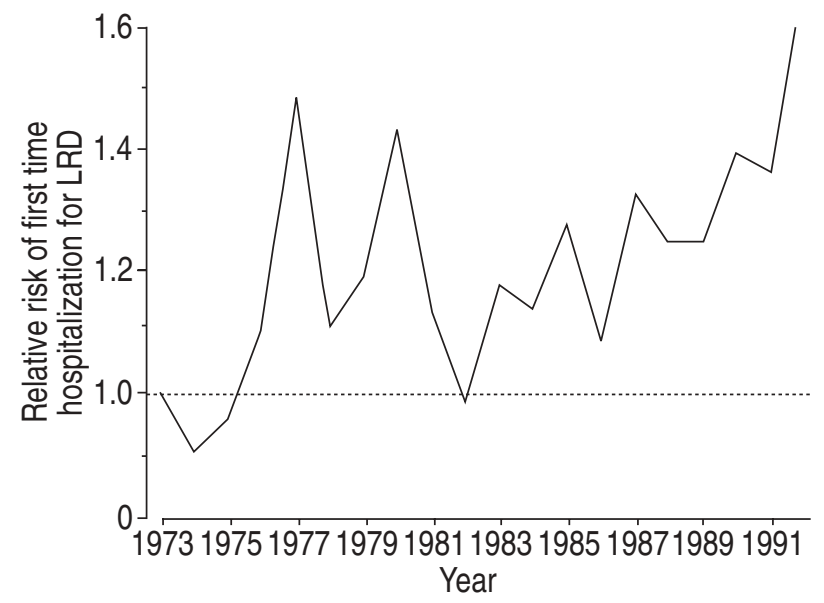

Fig. 1. - Relative risk of first time hospital admittance for lower respiratory disease (LRD) among children aged 0-4 yrs of age during 19731992 in Stockholm County. The year 1973 was used as the reference value (...).

The average increase per year was $1.82 \%$ (95\% CI $1.52-$ $2.12 \%$ ) (fig. 1). The relative risk of LRD among boys compared to girls was 1.65 (1.60-1.70). Furthermore, a pronounced decrease in risk for hospitalization was seen with increasing age. Using the age group $0-1$ yrs as reference the RR values were $0.55(0.53-0.57), 0.26(0.25-0.27)$, $0.14(0.13-0.15)$ and $0.1(0.09-0.11)$ in the age categories $1-2,2-3,3-4$ and $4-5$ yrs, respectively. Adding lower respiratory diseases without diagnosed pulmonary obstruction increased the study material by $10.4 \%$, and this proportion tended to decrease at the end of the study period.

Some peaks in hospitalization rate in the younger age groups coincide with outbreaks of RSV infections (fig. 2). The increasing time-trend was evident especially for the younger children. For children aged $<1$ yr, the overall increase was $100 \%$ during the observation period. From 1982-1992 the most pronounced gradual increase of first time admittance for LRD was observed. During 19821987 an increase of the re-admission rate for LRD was observed, but this rate tended to decrease during the last 5 yrs of the study period, in spite of the rise in first time admissions (fig. 3).

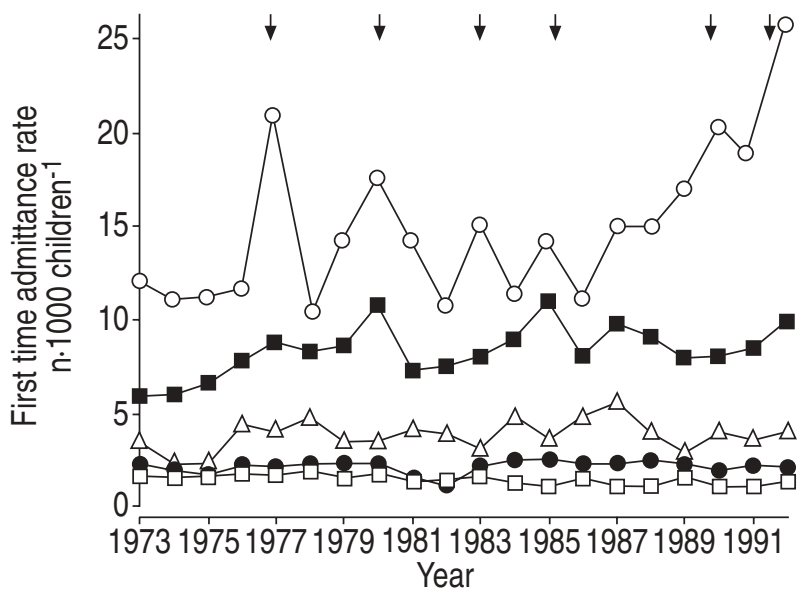

Fig. 2. - Rate of first time admittance for lower respiratory disease in children aged 0-4 yrs, during 1973-1992, in Stockholm County. The arrows indicate years with epidemic outbreaks of respiratory syncytial virus. $\bigcirc$ : children aged $<1 \mathrm{yr} ; \boldsymbol{\square}: 1-2 \mathrm{yrs} ; \Delta: 2-3 \mathrm{yrs} ; \bullet: 3-4 \mathrm{yrs} ; \square: 4-$ 5 yrs. 


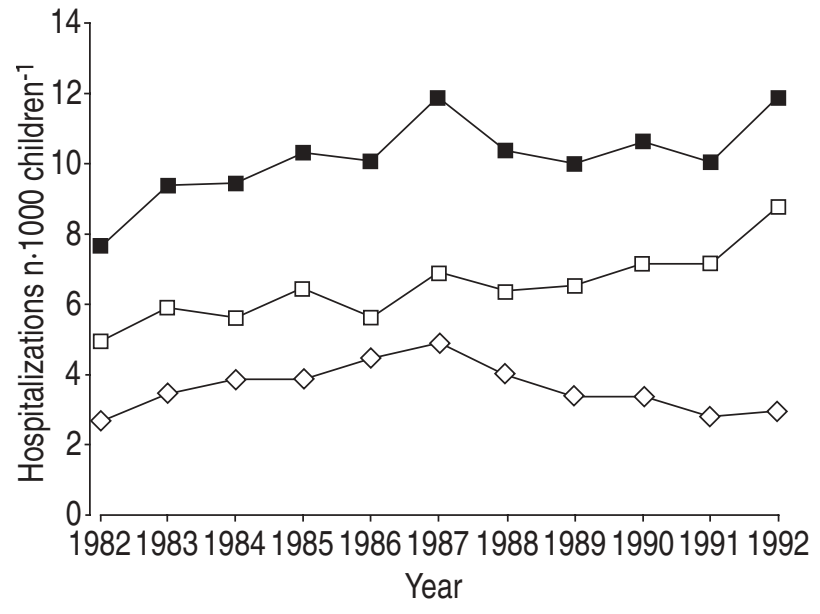

Fig. 3. - Population based hospital admission rate for lower respiratory disease (LRD) during 1982-1992 among children aged $<5$ yrs in Stockholm County. : total rate of hospitalization for LRD; $\square$ : first-time hospitalization for LRD; $\diamond:$ re-hospitalization for LRD.

During 1982 and 1992 the rates of first time hospitalization for LRD differed between the 25 municipalities in Stockholm County (fig. 4). In municipalities belonging to the referral area of two of the four paediatric clinics (Department of Pediatrics at Huddinge and Danderyd Hospitals), an increased rate of first time hospitalization for LRD was found during the study period, but this was not seen in the areas served by the other two hospitals. No association was found at municipal level between the rate of admittance of LRD and average income, residential density, educational level or number of immigrants.

From 1982 to 1992 the total number of admissions per 1,000 children, excluding admissions for LRD, decreased by $30 \%$ at the four children's hospitals (data not shown). The number of available beds decreased by an average of about $15 \%$ during this period. Furthermore, there was a

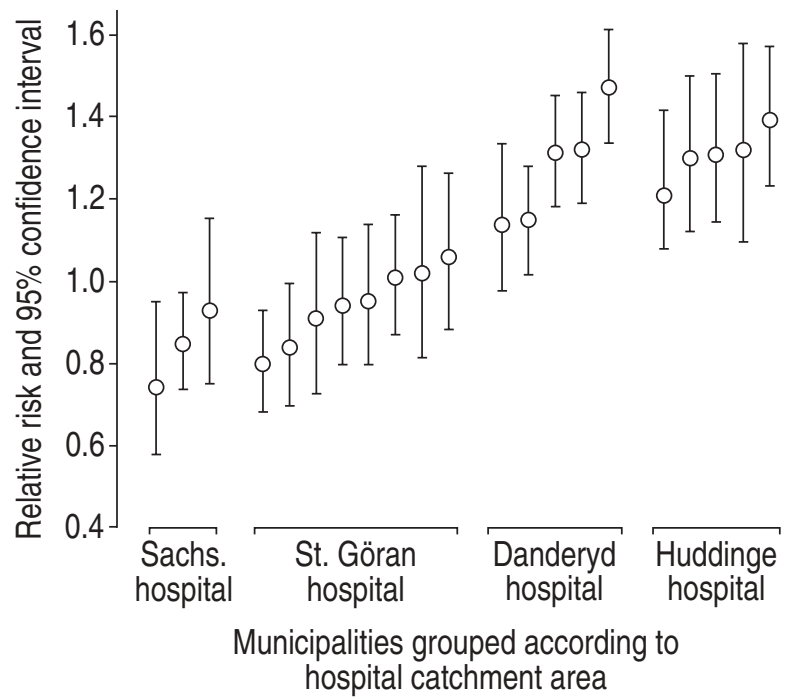

Fig. 4. - Relative risks of first time admittance for lower respiratory disease in children in relation to municipality and catchment area of the four children's hospitals in Stockholm County. The Stockholm inner city municipality constitutes the reference category as children in this district can be admitted to either of the four children's hospitals in Stockholm county. Values are mean $\pm 95 \%$ confidence interval. Sachs.: Sasska. reduction of the mean duration of hospitalization, which was noted especially for children aged $<1 \mathrm{yr}$. In this age group the mean duration of hospitalization decreased from 4.6 to 2.7 days (41\%). However, despite differences in accessibility of beds between the four paediatric hospitals, the time trend of first time hospitalization for LRD was consistent during the entire study period.

\section{Discussion}

In this survey we studied first time admittance for asthma, bronchitis and viral pneumonia in young children, as there is no clear distinction between the conditions, in particular among infants aged $<2$ yrs. During the study period of 20 yrs there was a $40 \%$ increase in the population based hospitalization rate for LRD in children aged $<5$ yrs with a marked increase $(100 \%)$ in the youngest age group. However, among the children aged $2-4$ yrs no increase of first time admittance for LRD was observed. Children aged $<2$ yrs predominated, which has also been described by others [5].

During recent decades a marked increase of hospitalization for asthma in infants has been reported from other countries [3]. In a study from the South West Thames Region, UK, admissions for childhood asthma increased by $186 \%$ during $1978-1985$ among children aged up to 5 yrs [4]. In a study from Norway with data from the 1980s a more than twofold increase in the hospitalization rate was recorded for children aged $<3$ yrs [5]. The observed increase in first time admission observed in our study should be compared to a total decrease of admissions (including re-admissions) for asthma and obstructive bronchitis as reported by others in Sweden $[25,26]$. In these studies no distinction was made between first time hospitalization and re-admission $[4,5]$. Furthermore, the results from these studies were not adjusted for population density in each age strata and gender. Our data showing a gradual increase in first time admissions and a decrease of re-admissions for LRD seen after 1987, correspond with those of others who report that admissions to hospital of children with asthma may have reached a plateau or even fallen $[5,6,25,26]$. The decrease of re-admissions for childhood asthma reported from Sweden is in agreement with our data indicating a decrease of re-admissions after 1987 for LRD and has been suggested to reflect changes in medical care and utilization of services [7, 26]. Our results are likely to reflect a true increase, as both readmissions for LRD and total admissions to hospital for paediatric internal diseases decreased during the latter part of the study period, with the most pronounced increase being in first time admissions for LRD.

In children aged $<3$ yrs, LRD is often caused by viral infections $[2,9]$. In our study a coincidence was observed between epidemic outbreaks of RSV infections and first time hospitalization for LRD in this age group. An increased incidence of RSV bronchiolitis could have contributed to the overall time trend of first time hospitalization for LRD during the study period. However, data are not available for detailed assessment of the RSV-positive cases. Breast-feeding has been reported to provide some protection against obstructive disease in childhood [19-21]. On the other hand, exposure of environmental tobacco smoke seems to increase the risk [14]. During 1983-1991 
reported breast-feeding in Stockholm County of babies aged up to 4 months increased from 65 to $74 \%$ (unpublished data, Child Health Centres of Stockholm County Council) with a simultaneous drop in reported smoking among pregnant women from 32 to $24 \%$ (unpublished data, Swedish Medical Birth Register). These trends in breast-feeding and smoking habits among pregnant women were not reflected in the trend of hospitalization for LRD, which indicates that other risk factors are also involved.

The increasing rate of first time hospitalization for LRD was confined to the youngest age groups. The absence of an increase among the children aged 2-4 yrs may have several explanations, including changes in utilization of health services over time [6] and, changes in pharmacological treatment plans [27], such as extended use of inhaled steroids [6], factors that may all reduce the severity of pulmonary obstructive disease. In accordance with international treatment guidelines, children aged $<2$ yrs are rarely given regular treatment with inhaled steroids at the onset of pulmonary obstructive disease. The decrease in re-admissions seen after 1987 may be explained by the extended use of anti-inflammatory treatment, i.e. inhaled steroids, that has been reported by others $[25,26]$. Changes in doctors' attitude towards diagnosis and treatment of the disorder and parents' attitudes towards their children's health may also affect the admission rate. However, this was not assessed in the present study.

The increase in the hospitalization rate seen especially after 1987 could have several explanations [6]. The change of the ICD codes in 1987 may have influenced the hospitalization rate during the following years, but probably did not affect the further increase seen in 1990-1992. The primary health care services in Sweden expanded during the 1980 s. This coincides with a $15 \%$ reduction in the number of beds at the paediatric clinics (official data, Stockholm County Council) and a simultaneous reduction of patient days as well as a marked reduction in total paediatric admissions. Overall, the accessibility for hospitalization may have changed and this could have contributed to the decrease in hospitalization at the beginning of the 1980s, but it does not explain the increase thereafter.

At two of the hospitals, the rate of first time hospitalization for LRD was higher than in the other two. Hypothetically, this may be explained by differences in priorities of hospitalization of children at first time of admittance for LRD or by regional differences in incidence. However, a similar increase of the hospitalization rate for LRD occurred at all four children's hospitals within Stockholm County during the study period. In-patient capacity may also have been a major factor as the two hospitals with the highest rates of first time admission for LRD experienced smaller reductions in the number of beds than the other two clinics. The in-patient days for asthma and obstructive bronchitis decreased during the study period, which may indicate that the severity of LRD has decreased and/or that medical therapy improved. It would have been interesting to follow children admitted to the emergency ward but not hospitalized, and compare them with the children hospitalized for LRD. This was not possible as only the hospitalized children were registered. During hospitalization, pharmacological treatment can be individualized, information and instructions given on drug inhalation techniques, written medication plan distributed and the importance of irritant and allergen avoidance mentioned [28]. All these issues are critical for the prognosis of children with obstructive LRD, as wheezing and RSV bronchiolitis in early childhood increases the risk for asthma later in life [2, 29].

In conclusion, we studied first time population based hospitalization for lower respiratory disease, including pulmonary obstructive respiratory disease, in children aged $<5$ yrs. During the study period of 20 yrs there was an average yearly increase in the hospitalization rate of $1.82 \%$, with a concomitant decrease of re-admissions during the last 5 yrs of the study period. The increase in hospitalization rate was primarily attributable to children aged up to $3 \mathrm{yrs}$. Peaks in hospitalization seemed to coincide with outbreaks of respiratory syncytial virus infections and boys were hospitalized more often than girls. Several factors besides incidence may influence the hospitalization rate for lower respiratory disease, such as changes in severity, medical guidelines, access to hospital care, in-patient capacity, hospital policy, attitude of doctors towards diagnosis and treatment, expansion of primary health care and attitudes of parents towards their children. The use of admittance for obstructive lower respiratory disease, even when restricted to first time admittance, as a surrogate for the incidence of asthma needs to be questioned, especially in young children.

\section{References}

1. Anderson LJ, Parker RA, Strikas RA, et al. Day-care center attendance and hospitalization for lower respiratory tract illness. Pediatrics 1988; 82: 300-308.

2. Wennergren G, Åmark M, Åmark K, Óskarsdóttir S, Sten $\mathrm{G}$, Redfors $\mathrm{S}$. Wheezing bronchitis re-investigated at the age of 10 years. Acta Paediatr 1997; 86: 351-355.

3. Mitchell EA. International trends in hospital admission rates for asthma. Arch Dis Child 1985; 60: 376-378.

4. Anderson HR. Increase in hospital admissions for childhood asthma: trends in referral, severity, and readmissions from 1970 to 1985 in a health region of the United Kingdom. Thorax 1989; 44: 614-619.

5. Carlsen K-H. Epidemiology of childhood asthma. Eur Respir Rev 1994; 4: 5-9.

6. Anderson HR, Butland BK, Strachan DP. Trends and prevalences and severity of childhood asthma. $\mathrm{Br}$ Med $\mathrm{J}$ 1994; 308: 1591-1596.

7. Carlsen KH, Örstavik I, Leegard J, Hoeg H. Respiratory virus infections and aeroallergens in acute bronchial asthma. Arch Dis Child 1984; 59: 310-315.

8. Leibovitz E, Freihorst J, Piedra PA, Ogra PL. Modulation of systemic and mucosal immune response to inhaled ragweed antigen in experimentally induced RSV infection. Int Arch Allergy Appl Immunol 1988; 86: 112-116.

9. Balfour-Lynn IM. Why does viruses make infants wheeze? Arch Dis Child 1996; 74: 251-259.

10. Wickman M, Nordvall SL, Pershagen G. Risk factors in early childhood for sensitization to airborne allergens. Pediatr Allergy Immunol 1992; 3: 128-132.

11. Palmieri M, Longobardi G, Napolitano G, Simonetti DM. Parental smoking and asthma in childhood. Eur J Pediatr 1990; 149: 738-740.

12. Willers S, Svennonius E, Skarping G. Passive smoking and childhood asthma. Urinary cotinine levels in children with asthma and in referents. Allergy 1991; 46: 330-334. 
13. Wright A, Holberg C, Martinez F, Taussig LM. Relationship and parental smoking to wheezing and non-wheezing, lower tract respiratory illness in infancy. $J$ Pediatr 1991; 118: 207-214.

14. Rylander E, Pershagen G, Eriksson M, Nordvall L. Parental smoking and other risk factors for wheezing bronchitis in children. Eur J Epidemiol 1993; 9: 517-526.

15. Strachan DP. Damp housing and childhood asthma: validation of reporting of symptoms. BMJ 1988; 297: 12231226.

16. Andrae S, Axelson O, Björkstén B, Fredriksson M, Kjellman NIM. Symptoms of bronchial hyperreactivity and asthma in relation to environmental factors. Arch Dis Child 1988; 63: 473-478.

17. Wickman M, Nordvall L, Pershagen G, Sundell J, Schwartz B. House dust mite sensitization in children and residential characteristics in a temperate region. J All Clin Immunol 1991; 88: 89-95.

18. Horwood LJ, Ferguson DM, Hons BA, Shannon FT. Social and familial factors in the development of early childhood asthma. Pediatrics 1985; 75: 859-868.

19. Wright A, Holberg C, Martinez FD, et al. Breastfeeding and lower respiratory tract illness in the first years of life. Br Med J 1989; 29: 946-949.

20. Arshad SH, Matthews S, Gant C, Hide DW. Effect of allergen avoidance on development of allergic disorders in infancy. Lancet 1992; 339: 1493-1497.
21. Infante-Rivard C. Childhood asthma and indoor environmental risk factors. Am J Epidemiol 1993; 8: 834-844.

22. Burr ML, Limb ES, Maguire MJ, et al. Infant feeding, wheezing, and allergy: a prospective study. Arch Dis Child 1993; 68: 724-728.

23. Breslow NW, Day NE. Indirect standardization and multiplicative models for rates, with reference to the age adjustment of cancer incidence and relative frequency data. J Chronic Dis 1975; 28: 289-303.

24. GLIM statistical package. Royal Statistical Society, London 1985.

25. Wennergren G, Kristjánsson S, Strannergård I-L. Decrease in hospitalisation for treatment of childhood asthma with increased use of antiinflammatory treatment, despite an increase in prevalence of asthma. J Allergy Clin Immunol 1996; 97: 742-748.

26. Ströberg L. Decreasing admissions for childhood asthma to a Swedish county hospital. Acta Paediatr 1996; 85: 173-176.

27. Connett G, Lenny W. Prevention of viral induced asthma attacks using inhaled budesonide. Arch Dis Child 1993; 68: 85-87.

28. Mellis CM, Peat JK, Woolcock AJ. The costs of asthma. Can it be reduced? Pharmacoeconomics 1993; 3: 205-219.

29. Pullan CR, Hey EN. Wheezing, asthma, and pulmonary dysfunction 10 years after infection with respiratory syncytial virus in infancy. Br Med J 1982; 284: 1665-1669. 